\title{
Meningiomas of the ventral foramen magnum
}

\author{
Kenan I. Arnautovic, M.D., Ossama Al-Mefty, M.D., F.A.C.S., and Muhamed Husain, M.D. \\ Departments of Neurosurgery and Pathology, University of Arkansas for Medical Sciences, and \\ Laboratory Service, Veterans Administration Medical Center, Little Rock, Arkansas
}

Meningiomas of the ventral foramen magnum are rare lesions that account for less than $3 \%$ of all meningiomas. Their treatment remains one of the most challenging among all meningiomas. The authors comprehensively analyzed multiple features in the series of patients who harbored these lesions.

The authors conducted a retrospective study of 18 patients who harbored lesions in the ventral foramen magnum (mean follow up 40 months) in whom surgery was performed via a transcondylar approach. Sixteen patients underwent surgery for the first time: in 12 patients $(75 \%)$ gross total, in two $(12.5 \%)$ near total, and in two (12.5\%) subtotal tumor removal was achieved. The remaining two patients were treated for a recurrent tumor. Karnofsky Performance Scale (KPS) scores obtained at follow-up review demonstrated statistically significant improvement compared with those obtained preoperatively. The extent of surgical resection and preoperative KPS score were variables that demonstrated statistically significant favorable influence on outcomes. Ninth and 10th cranial nerve palsies were the most common complication that contributed to the prolonged hospital stay; six patients who experienced nerve palsy preoperatively worsened postoperatively, and four other patients developed nerve palsy after surgery. There were no perioperative deaths. Four patients died during the follow-up period. One patient died of multiple myeloma; another, who underwent surgery for a recurrent tumor, died 3 years after this second surgery from new tumor recurrence at 80 years of age; and the remaining two patients died 1.5 and 5 months postsurgery of pulmonary embolus and endocarditis, respectively.

Meningioma of the ventral foramen magnum can be radically removed in a majority of patients in whom complications will be frequent but transient, resulting from lower cranial nerve palsies. The radical removal of a recurrent tumor provides for the patient a relatively long, stable follow-up period. Patients presenting with a low KPS score have a poor prognosis. Early diagnosis and treatment are recommended.

Key Words * foramen magnum * meningioma * outcome * skull base surgery * transcondylar approach

Meningiomas of the foramen magnum arise at the dura of the craniocervical junction. The zone from which they arise corresponds anteriorly to the area between the lower third of the clivus and the upper edge of the axis, laterally from the jugular tubercle to the upper aspect of the C-2 lamina, and posteriorly 
from the anterior edge of the squamous occipital bone to the C-2 spinous process.[15-17] They represent between 0.3 to $3.2 \%$ of all meningiomas, 4.2 to $20 \%$ of all meningiomas of the posterior fossa, and 60 to $77 \%$ of all benign extramedullary tumors at the craniospinal

junction.[3,10,12,15,19,23,27,29-31,36,39,40]

We defined as ventral foramen magnum meningiomas those meningiomas that arise anterior to the ascending coronal plane between the first dentate ligament and the ninth through 12th cranial nerves on both sides. They occur in 68 to $98 \%$ of all meningiomas of the foramen magnum[10,15,17,26,30,31,35] and should be clearly distinguished from meningiomas of the dorsal (posterior) foramen magnum because of the remarkable differences in presenting symptoms, neurological findings, operative approach, postoperative results, complications, and mortality rate. Meningiomas of the ventral foramen magnum are one of the most challenging types of meningiomas to treat because a large number of vital neurovascular structures, which are sensitive to injury, are crowded in this deeply hidden central area. These neurovascular elements drape the tumor, making its radical removal both difficult and associated with a relatively high rate of complications. However, the development of modern diagnostic techniques, the refinement of microsurgical techniques, the microsurgical anatomical studies, the development of skull base approaches, the advancements in neuroanesthesia, intraoperative neurophysiological monitoring and careful multidisciplinary perioperative planning have advanced the treatment of these difficult lesions, allowing for more radical tumor resection, decreasing the rate of complications, and improving the patient survival.[1-7,13,15-26,29,31,34,36-41]

We had the privilege of treating, over a relatively short period of time and with a consistent protocol, a series of patients who harbored these lesions. In this paper, we describe our experiences, complications, pitfalls, and lessons learned from this series. In addition, we present the perioperative management protocol that we developed and a brief histological review of the relevance of our series.

\section{CLINICAL MATERIAL AND METHODS}

We retrospectively analyzed our series of 18 patients in whom surgery was performed by a senior author (O.A.) over an 8.25-year period (December 1990 through April 1999). Eighteen patients with meningioma of the ventral foramen magnum were treated surgically in three neurosurgical departments at the University of Mississippi Medical Center (1990), Loyola University Medical Center (1991-1993), and the University of Arkansas for Medical Sciences (1993-1998). Our study excluded patients with meningioma located dorsally in the foramen magnum or those originating elsewhere and extending into the foramen magnum (for example, the jugular foramen, upper clivus, cerebellopontine angle, and the spinal canal). We also excluded patients in whom malignant or aggressive meningioma types were diagnosed. Data from each patient's medical chart, follow-up examination, and neuroradiology and histological studies were reviewed. For patients who could not be followed on a regular basis, we obtained medical and radiological reports or contacted the patients directly through phone calls and questionnaires. The Karnofsky Performance Scale (KPS)[21] was used to evaluate the patient's clinical course. The differences between the preoperative and a follow-up KPS scores were examined by conducting a paired t-test. With regard to clinical outcomes, Cox's proportional hazard model was used to test the influence of variables such as age, sex, preoperative KPS score, and the extent of tumor resection.

The degree of tumor resection was classified based on the surgeon's observation detailed in the operative report and on postoperative neuroradiological findings. If these elements did not agree, we relied on the 
postoperative images. We divided the degree of tumor resection into three categories: gross-total resection (excision of the dural attachment and drilling of adjacent bone [Simpson Grades I and II]); near-total resection (in which a few millimeters of insulated and cauterized tumor were left on the vertebral artery [VA] or other vital structure if the arachnoidal plane could not be established [Simpson Grade III]); and subtotal removal of more than 50\% of the tumor mass (Simpson Grade IV).[34]

The portion of condyle that was drilled ranged from one-third to one-half. The amount of drilling was directly proportional to the tumor location and its extension to the contralateral side to allow safer dissection from vital anatomical structures. In other words, the more medial the tumor or the more it extended across the midline, the more the condyle was drilled. In all but three patients the tumor was removed in a single sitting. The complex venous compartment in the suboccipital area that cushions the VA complex was previously named the suboccipital cavernous sinus (SCS). $[3,4,41]$ In these patients, dissection of the SCS and the transposition of the VA complex were difficult. Therefore, the intradural part of surgery was postponed 1, 2, and 5 days, respectively. In one patient, the tumor adhered to the VA and the 11th cranial nerve, and an arachnoidal plane could not be established. Our persistence in separating these structures led to rupture of the VA, which was repaired by microsurgical sutures and reinforced with a Sundt clip. The 11th cranial nerve was transected, dissected free, and reanastomosed.

\section{Perioperative Protocol}

The perioperative protocol was developed over the years of our experience, and it was also based on the experiences of others in treating meningioma of the foramen magnum,[5-12,14-20,22,32,33,35-40] general perioperative skull base management principles, and lessons learned from each case. Treatment was tailored to the needs of each patient. Following is a brief outline of the important features of the protocol.

Neuroradiologic Work Up. Each patient underwent a battery of neuroradiological tests. Magnetic resonance (MR) imaging included views in axial, coronal, and sagittal planes, and multiple relaxation techniques were included such as $\mathrm{T}_{1}$ and $\mathrm{T}_{2}$-weighted and proton density images. This workup included a 1-mm-thick slicing protocol for image-guided frameless stereotaxy; MR imaging was performed more often than computerized tomography (CT) for this purpose. Magnetic resonance angiography was performed simultaneously and included both arteriographic and venous phases. We abandoned the routine preoperative use of four-vessel angiography unless MR angiography study provided particularly unusual findings that required further evaluation. The patency of the vertebral and basilar arteries, venous sinuses, their dominance and positions of jugular bulbs were particularly important. Computerized tomography studies included bone-window scans. The CT imaging protocol included 1-mm-thick slices and views in all three planes to evaluate the area of tumor attachment and features of the condyle and C1-2 anatomy, among other data. Postoperative imaging studies included CT scanning within 24 hours postsurgery and MR imaging within several days. These studies were used to evaluate the extent of tumor removal and possible complications, and they also served as a baseline. Chest x-ray films were obtained every day after surgery as a baseline and to rule out aspiration, if necessary. Follow-up evaluation included MR imaging with and without injection of contrast medium and was scheduled 3, 6, and 12 months after discharge. These evaluations were followed by yearly examinations, as needed. For patients in whom subtotal resection was performed, we considered adjunctive treatment, such as radiation therapy or radiosurgery (two and two, respectively).

Multidisciplinary Evaluations. Regardless of the preoperative function of the ninth through 12th cranial 
nerves, a speech pathologist evaluated each patient. Evaluation included swallowing studies (for example, bedside and barium swallow) both for an assessment and as a baseline. These studies were coupled with otolaryngological evaluation if a patient's status required it. Postoperatively, a patient was maintained on a "nothing per mouth" regimen until cleared by a speech pathologist. If a patient experienced swallowing difficulties, appropriate actions (parenteral nutrition, an orogastric or a gastric tube, vocal cord injections and medialization, and tracheostomy) were undertaken as needed. Depending on the extent of the tumor, ophthalmological, audiometrical, electromyographic monitoring, and other necessary studies were added. Aggressive respiratory management, physical and occupational therapy, and consultations for rehabilitation were instituted as necessary early in the postoperative period.

Anesthetic Management. With the patient awake, endoscopic intubation was performed routinely to avoid hyperextension of the neck. Systemic monitoring included an arterial line (normotension is strictly monitored), a central venous line, a Foley catheter, a Doppler monitor, electrocardiography, and an oxymeter. A loading dose of steroids was administered when anesthesia was induced. Recently the use of a methylprednisolone protocol in cases of spinal cord injury was initiated. Initial doses of antibiotics (usually vancomycin and ceftazidime) were also introduced at that time and typically discontinued after 72 hours.

Intraoperative Neurophysiologic Monitoring. Somatosensory evoked potentials and brain auditory evoked potentials were monitored bilaterally. The 10th cranial nerve was monitored through an electromyography endotracheal tube. The 11 th and 12 th cranial nerves were also monitored, unilaterally or bilaterally, as needed.

\section{Operative Technique}

We used the transcondylar approach to resect all tumors in this series. The technique was tailored to each patient according to preoperative imaging, individual anatomy, and the tumor extension findings. If necessary, the approach can be extended or combined with other skull base approaches, and was required in two cases. This approach was greatly influenced by microsurgical anatomical studies of this complex area[4,13] and has been described in detail elsewhere.[1-4,20,36,37] We will provide a brief outline.

Patient Positioning. The patient was placed supine and rotated $40^{\circ}$. The head was kept in neutral position and fixed in a Mayfield headrest to which is attached an image-guided stereotactic frame. The left thigh and the abdominal wall were prepared for grafts of fascia lata or fat.

Skin Incision. A curvilinear skin incision was made 2 fingerbreadths behind the mastoid. It was begun at the height of the external acoustic meatus and continued downward to the level of the C-4 vertebra, gradually curving anteriorly.

Muscle Dissection. The muscles were dissected in three layers. As a rule, to prevent obscuring of the surgeon's axis, which was mainly lateral, all muscles were reflected inferiorly and medially after detachment. First, the sternocleidomastoid was detached from its origin at the occipital bone, which carefully preserved the innervation of the 11th cranial nerve. The digastric groove with the digastric muscle was an important landmark for localization of the facial nerve. Second, the splenius capitis, longissimus capitis, and semispinalis muscles were detached and retracted. The rich, suboccipital venous plexus was found between the second and third muscular layers. This plexus may be the source of bleeding or air embolism, as can be the mastoid emissary vein as it exits through the mastoid emissary foramen. The third muscular layer consisted of six small muscles that delineate two suboccipital 
triangles, the superior and inferior.[4] In the depth of the superior suboccipital triangle were the horizontal suboccipital VA and the $\mathrm{C}_{1}$ nerve cushioned in the venous compartment, the SCS.[3,4,41] In the depth of the inferior suboccipital triangle were the vertical suboccipital VA and the $\mathrm{C}_{2}$ nerve surrounded by the VA venous plexus. Both triangles met at the transverse process of the atlas, $1 \mathrm{~cm}$ below the mastoid. After the muscles were reflected, the posterior atlantooccipital membrane was opened.

Exposure, Mobilization, and Transposition of the VA Complex. From this point on, we focused the extradural part of the transcondylar approach on the suboccipital VA in its venous compartment (SCS). The transverse foramen of the atlas was drilled open, and the lateral (periosteal) ring around the VA complex at its superolateral loop was dissected subperiosteally. The posterior and lateral condylar veins were divided, which preserved the wall ("periosteal sheet") around the SCS (Fig. 1). The retrocondylar ligament was also divided, as were the arterial branches of the horizontal suboccipital VA. The VA complex was then carefully transposed inferiorly and medially. The sinus can be the source of significant bleeding, which can be controlled with Gelfoam and gentle compression.

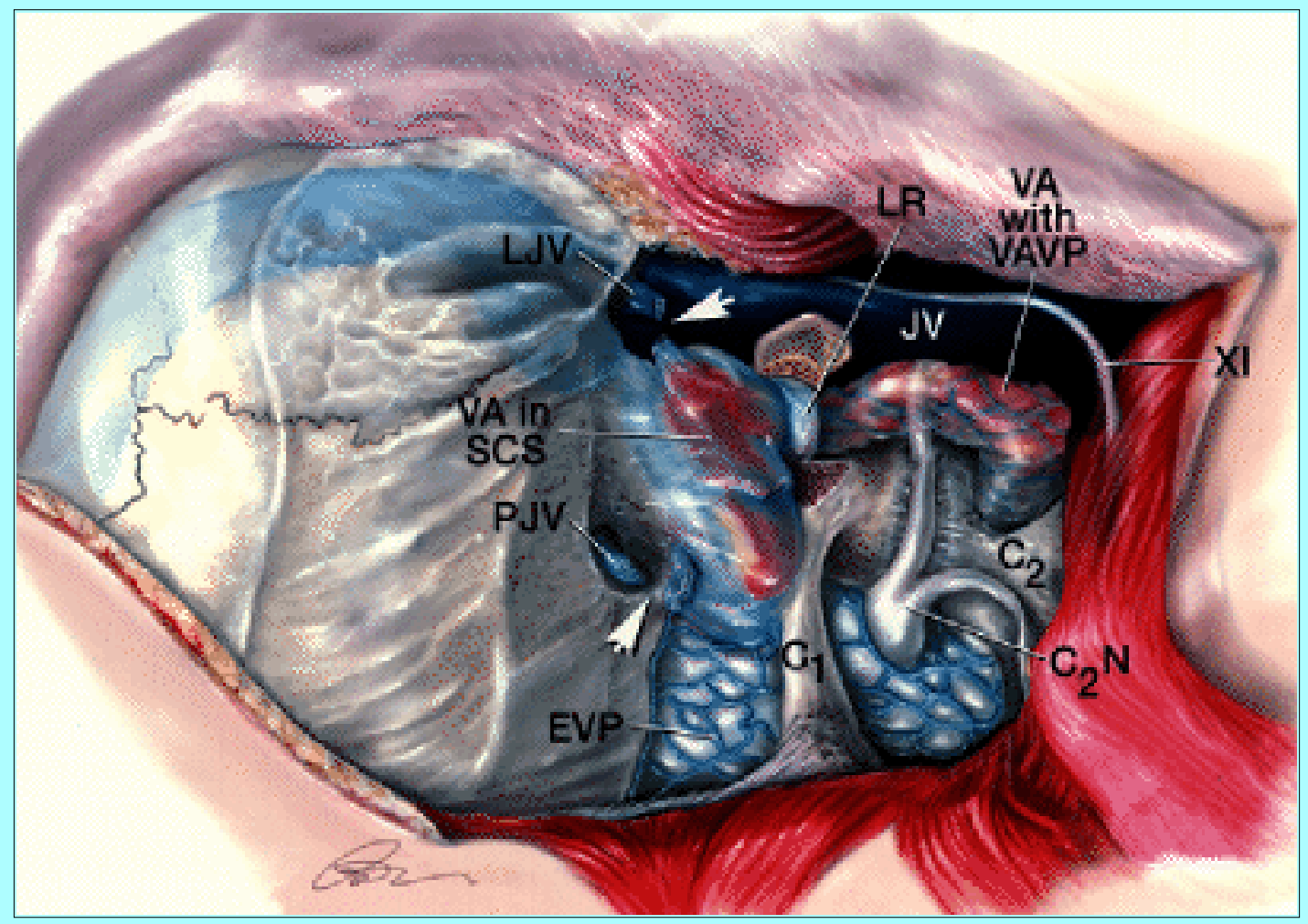

Fig. 1. Artist's drawing of right-side intraoperative exposure obtained after the dissection and retraction of muscles and detachment of the posterior atlatooccipital membrane. The SCS is exposed, cushioning the horizontal part of the suboccipital VA. Note also the vertical part of the suboccipital VA surrounded by a VA venous plexus (VAVP), lateral (periosteal) ring (LR) after the drilling of the transverse foramen of $\mathrm{C}-1$, posterior (PCV) and lateral condylar veins (LCV) that are divided (arrow heads), the jugular vein ( $\mathrm{JV})$, the atlas $\left(\mathrm{C}_{1}\right)$, the second cervical nerve $\left(\mathrm{C}_{2} \mathrm{~N}\right)$, the axis $\left(\mathrm{C}_{2}\right)$, the external vertebral venous plexus $(\mathrm{EVP})$, and the 11th cranial nerve (XI) entering the sternocleidomastoid muscle.

Bony Resection. A lateral, posterior fossa craniotomy and mastoidectomy were performed. The sigmoid sinus and the jugular bulb were fully exposed. Drilling of the condyle was variable and depended on the size, location, and extension (across the midline or superior-inferior) of the tumor. In all cases, however, 
at least one-half of the condyle was preserved to maintain the stability of the joint. Laminectomy of the atlas and sometimes the axis was often performed.

Dural Opening. The dural incision was centered on the dural ring around the VA complex. The cuff around the dural ring was left large enough to permit later watertight closure. When the dura was opened, the posterior meningeal artery arising from the horizontal suboccipital VA was divided. This was actually the beginning of tumor devascularization.

Intradural Tumor Dissection. From this point on, further dissection was centered on the intracranial VA. In approximately half of the patients, the tumor completely encased the intracranial VA. In many cases, an arachnoidal plane, which allowed complete separation, was preserved between the vessel and the tumor. In some cases, however, this plane could not be established, and a few millimeters of tumor cuff were left around the intracranial VA. In these cases, persisting attempts at separating these structures may provoke rupture. In the case in which this occurred, we used microsurgical sutures and Sundt's circular enforcement clip. The posterior inferior cerebellar artery was usually displaced medially and dorsally; it may also be embedded by a tumor. The anterior and posterior spinal arteries usually adhered to the tumor, but a plane of dissection between the two could be established. The complex of cranial nerves (nine-12) was displaced posteriorly and at times split in a superior-inferior direction (Fig. 2).

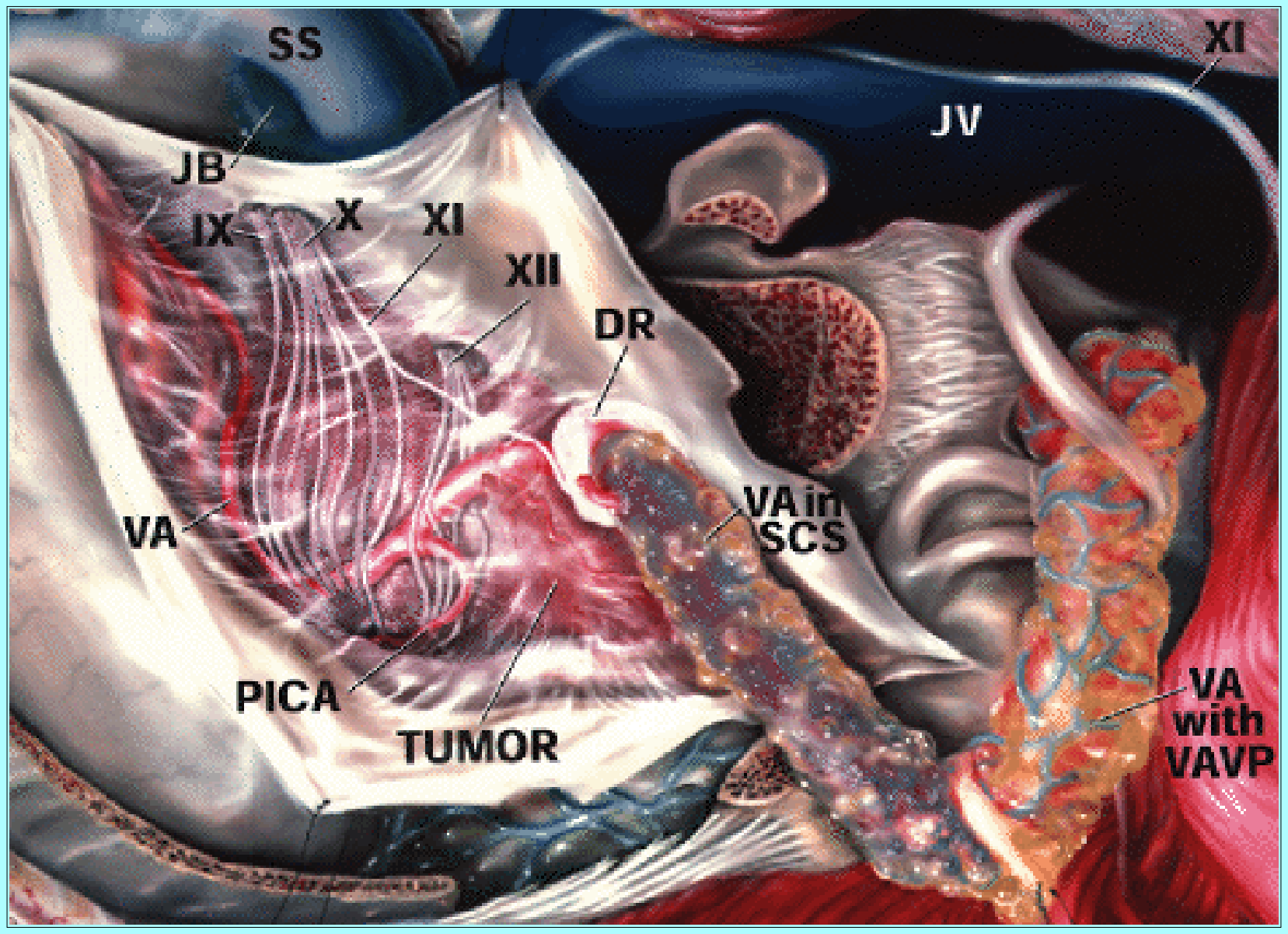

Fig. 2. Artist's drawing of the right-sided intraoperative exposure obtained after the VA complex transposition and the dural opening; the arachnoid membrane is still not opened. Note the transposed suboccipital VA complex (horizontal part) cushioned in the SCS, the vertical part of the suboccipital VA surrounded by the VA venous plexus, the posterior inferior cerebellar artery (PICA), the dural ring (DR) around the VA, ninth through 12th cranial nerves (IX-XII), the sigmoid sinus (SS), the jugular bulb (JB), and the jugular vein. 
See Fig. 1 for other abbreviations.

An arachnoidal plane nearly always existed between the tumor and the nerves. Displacement of the brainstem and spinal cord was posterior or to the contralateral side. The surgeon gained maneuverability by dividing the first dentate ligament and freeing the spinal part of the 11th cranial nerve along the arachnoidal planes. The dural attachment of tumor should be devascularized as soon as possible. The tumor was debulked with the ultrasonic aspirator, microsurgical technique, or both (Fig. 3).

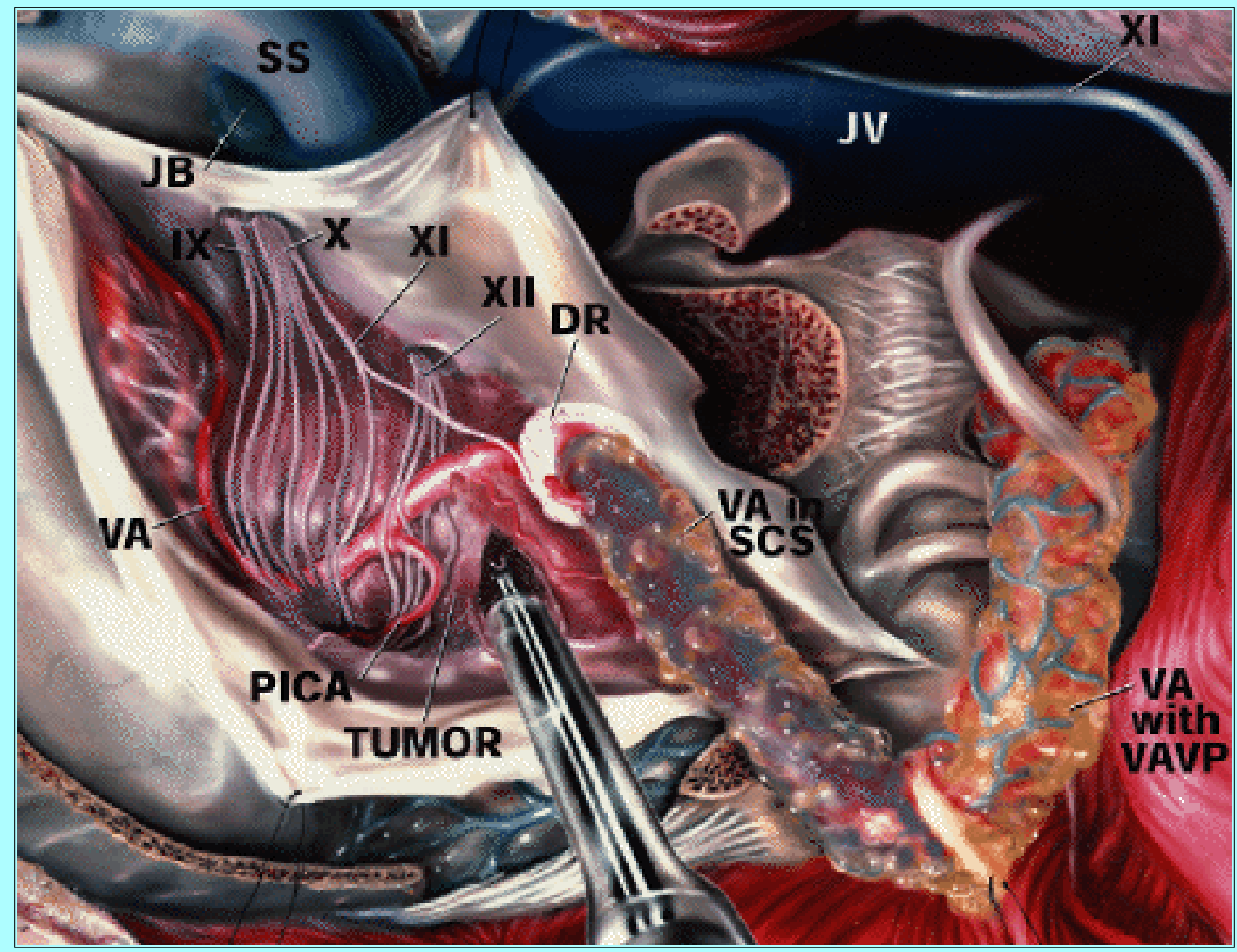

Fig. 3. Same drawing as seen in Fig. 2, after opening of the arachnoid membrane. The cavitron ultrasonic aspiration of the tumor is underway. See Figs. 1 and 2 for abbreviations.

Dura at the tumor attachment should be excised along with adjacent bone (that is, in resection classified as Simpson Grade I or II).[34] If part of the tumor was to be left behind, meticulous bipolar cauterization (and division) of the tumor attachment site and vascularization was performed. After the tumor was removed (Fig. 4), meticulous hemostasis and the Valsalva maneuver were ensured. 


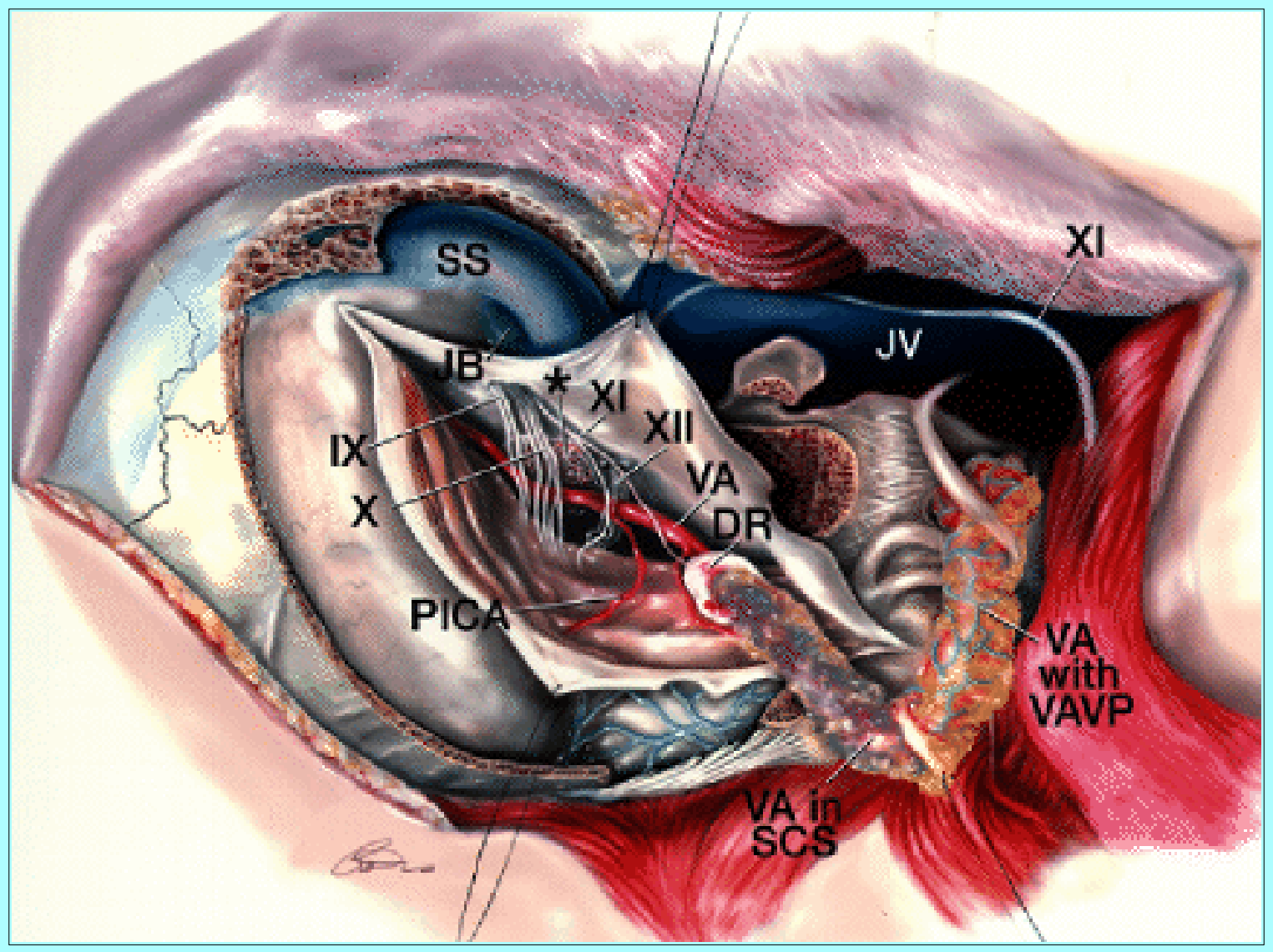

Fig. 4. Artist's drawing of the right-sided intraoperative exposure obtained after the tumor resection. Note the previous site of tumor attachment after the dural resection and the bone drilling $\left(^{*}\right)$, Simpson Grades I and II. Note the same anatomical elements as depicted in Figs. 2 and 3, now after the radical tumor resection.

Dural Closure. Dural closure in a watertight fashion was typically difficult when the VA complex was fully mobilized. However, it should be done with zeal by using of an autologous fascia lata, a fat graft, and fibrin glue.

\section{RESULTS}

\section{Demographic Data}

In the series of 18 patients who harbored meningioma in the ventral foramen magnum, ages ranged from 36 to 77 years (mean 58 years; Fig. 5). The most common age range was 45 to 64 years. The series was comprised of 13 women (72\%) and five men (28\%), a female/male ratio of 2.6:1. The average length of hospitalization was 18 days. Follow-up periods ranged from 0 to 84 months (mean 40 months). 


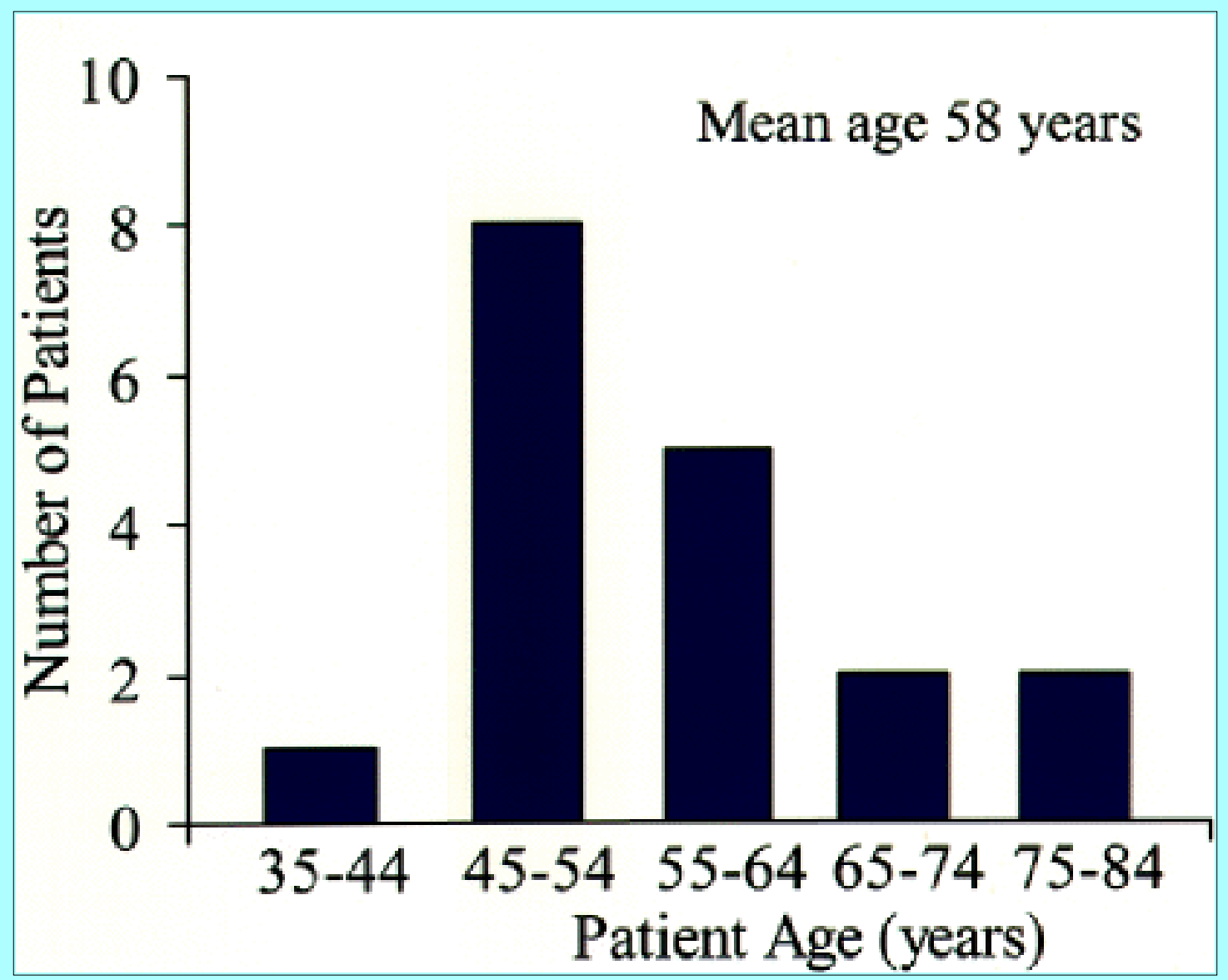

Fig. 5. Bar graph showing the age distribution of patients.

\section{Presenting Symptoms, Signs, and Findings}

The time between the first occurrence of symptoms and the surgery ranged from 1 month to 10 years (mean 45 months). The most common symptoms were occipital pain and headaches followed by ataxic incoordination, gait problems, and swallowing difficulties (Table 1). It was more common to observe a combination of two or more symptoms, signs, or findings than only one. One patient harbored a sizable ventrally located foramen magnum meningioma that was discovered incidentally. 


\section{TABLE 1}

PRESENT NG NEUROLOGICAL SIGNS, SYMPTOMS, AND FIND NGS IN 18 PATIENTS WHO HARBORED MEN NGLMA IN THE YENTRAL FORAMEN MAGNUM*

\begin{tabular}{|c|c|}
\hline Characteristic & $\begin{array}{c}\text { No. of } \\
\text { Patients (\%) }\end{array}$ \\
\hline \multicolumn{2}{|l|}{ painsensory changes } \\
\hline occipital painheadache & $13(72)$ \\
\hline facial num bness pain & $4(22)$ \\
\hline hand numbness & $4(22)$ \\
\hline hemihypesthesia & $1(6)$ \\
\hline leg num bness & $1(6)$ \\
\hline decreased hearing (CN VIII) & $1(6)$ \\
\hline \multicolumn{2}{|l|}{ cranial ner'e deficits (Motor) } \\
\hline swallo wing difficulties (CN $I X, X$ ) & $6(33)$ \\
\hline diplopia (CN VI) & $5(28)$ \\
\hline shoulder meakness (CN XI) & $3(17)$ \\
\hline tongue meakness or spasms (CN XII) & $2(11)$ \\
\hline \multicolumn{2}{|l|}{ uncoordinationdoss of dexterity } \\
\hline ataxic Incoordination gait difficulties & $6(33)$ \\
\hline disarthria. & $1(6)$ \\
\hline hand dumsiness & $1(6)$ \\
\hline \multicolumn{2}{|l|}{ weakness } \\
\hline hemiparesis & $2(11)$ \\
\hline quadriparesis & $2(11)$ \\
\hline paraparesis & $1(6)$ \\
\hline hydrocephalus & $1(6)$ \\
\hline tumor found incidentally & $1(6)$ \\
\hline
\end{tabular}

\section{Surgical Data}

Of the 18 patients in our series, 16 underwent surgery for the first time whereas two patients, respectively, had undergone surgery 1 and 11 years earlier and had presented to us for treatment of recurrent tumors (Table 2). 


\begin{tabular}{|c|c|c|c|c|c|c|}
\hline \multicolumn{7}{|c|}{$\begin{array}{c}\text { TABLE } 2 \\
\text { CLINICAL, SURGKAL, AND OUTCOME DATA IN } 18 \text { PATIENTS WHO UNDERWENT SURGERV } \\
\text { FOR MEN NGOMA OF THE VENTRAL FORAMEN MAGNUM }\end{array}$} \\
\hline \multirow{2}{*}{$\begin{array}{l}\text { Case } \\
\text { No. }\end{array}$} & \multirow{2}{*}{$\begin{array}{l}\text { Age (ys) } \\
\text { Sex }\end{array}$} & \multirow[b]{2}{*}{ Resection } & \multicolumn{2}{|c|}{ KPS Score } & \multirow{2}{*}{$\begin{array}{c}F U \\
(m \infty)\end{array}$} & \multirow[b]{2}{*}{ Remarks } \\
\hline & & & Pre op & Postop & & \\
\hline 1 & $74, \mathrm{~F}$ & gross total & 70 & 100 & 44 & $\begin{array}{l}\text { died of multiple m yeloma } \\
\text { at } 62 \text { mos postop }\end{array}$ \\
\hline 2 & $62, F$ & subtotil & 60 & 70 & 32 & tumor stable \\
\hline 3 & $50, \mathrm{~F}$ & subtotal & 70 & 90 & 84 & tumor statle \\
\hline 4 & 77, M & subtotal & 70 & 90 & 35 & $\begin{array}{l}\text { op for recurrence; died } \\
37 \text { mos postop }\end{array}$ \\
\hline 5 & $73, \mathrm{~F}$ & gross total & 70 & 100 & 84 & \\
\hline 6 & $50, F$ & subtotal & 60 & 60 & 83 & $\begin{array}{l}\text { op for recurrence; tumor } \\
\text { stable }\end{array}$ \\
\hline $\begin{array}{l}7 \\
8\end{array}$ & $\begin{array}{l}53, F \\
56, M\end{array}$ & $\begin{array}{l}\text { gross total } \\
\text { gross total }\end{array}$ & $\begin{array}{l}80 \\
70\end{array}$ & $\begin{array}{r}100 \\
90\end{array}$ & $\begin{array}{l}75 \\
62\end{array}$ & \\
\hline 9 & $63, \mathrm{~F}$ & gross total & 30 & 40 & 4 & died 5 mos postop \\
\hline 10 & $36, M$ & near total & 90 & 100 & 54 & tumor stable \\
\hline 11 & $50, \mathrm{M}$ & near to tal & 70 & 90 & 47 & tumor stable \\
\hline 12 & $77, \mathrm{~F}$ & gross total & 40 & 40 & 1 & died 1.5 mos postop \\
\hline 13 & $53, \mathrm{~F}$ & gross total & 70 & 90 & 35 & \\
\hline 14 & 48, M & gross total & 80 & 90 & 33 & \\
\hline 15 & $63, \mathrm{~F}$ & gross total & 90 & 100 & 23 & \\
\hline 16 & $49, F$ & gross total & 80 & 100 & 15 & \\
\hline 17 & $56, F$ & gross total & 70 & 100 & 10 & \\
\hline 18 & $52, \mathrm{~F}$ & gross total & 90 & 90 & 0 & \\
\hline
\end{tabular}

Of the 16 patients in whom surgery was performed for the first time, 12 underwent gross-total, two near-total, and two subtotal removal of their tumors (Fig. 6). Both patients in whom surgery was performed for recurrent tumors underwent subtotal resection. In the four patients in whom subtotal resection was performed (two in the first-time, and two in the recurrent tumor group), the tumor tightly adhered to the brainstem, the vertebral and basilar arteries and their perforators (Table 2, Fig. 6). 


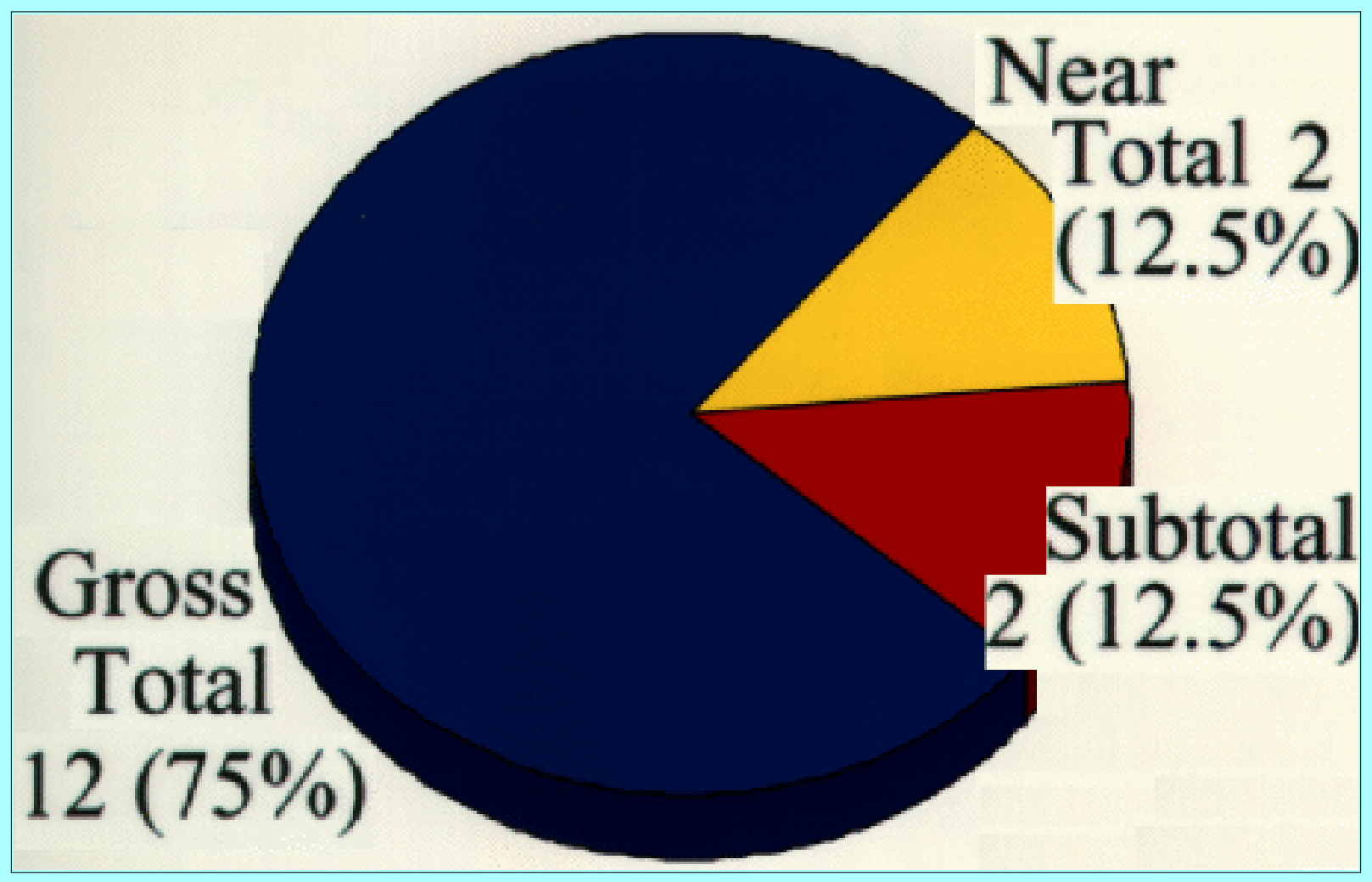

Fig. 6. Pie chart showing the extent of the tumor resection in 16 patients undergoing a first-time surgery for meningioma of the ventral foramen magnum.

In these patients, after maximum extent of tumor was resected, its origin was cauterized extensively, and the vascular supply was interrupted as much as possible. All four of these patients then underwent fractionated radiotherapy (two patients) or radiosurgery (two patients), and in three stable tumor control was demonstrated in the follow-up period (mean 5.5 years). In two patients (Cases 10 and 11; Table 2) there was an isolated, cauterized cuff of tumor a few millimeters thick and long around the VA (Fig. 7). An arachnoidal plane could not be established between the arterial wall and the tumor. After 54- (Case 10) and 48-month (Case 11) follow up (mean 51 months), their annual MR imaging studies demonstrated no change. 

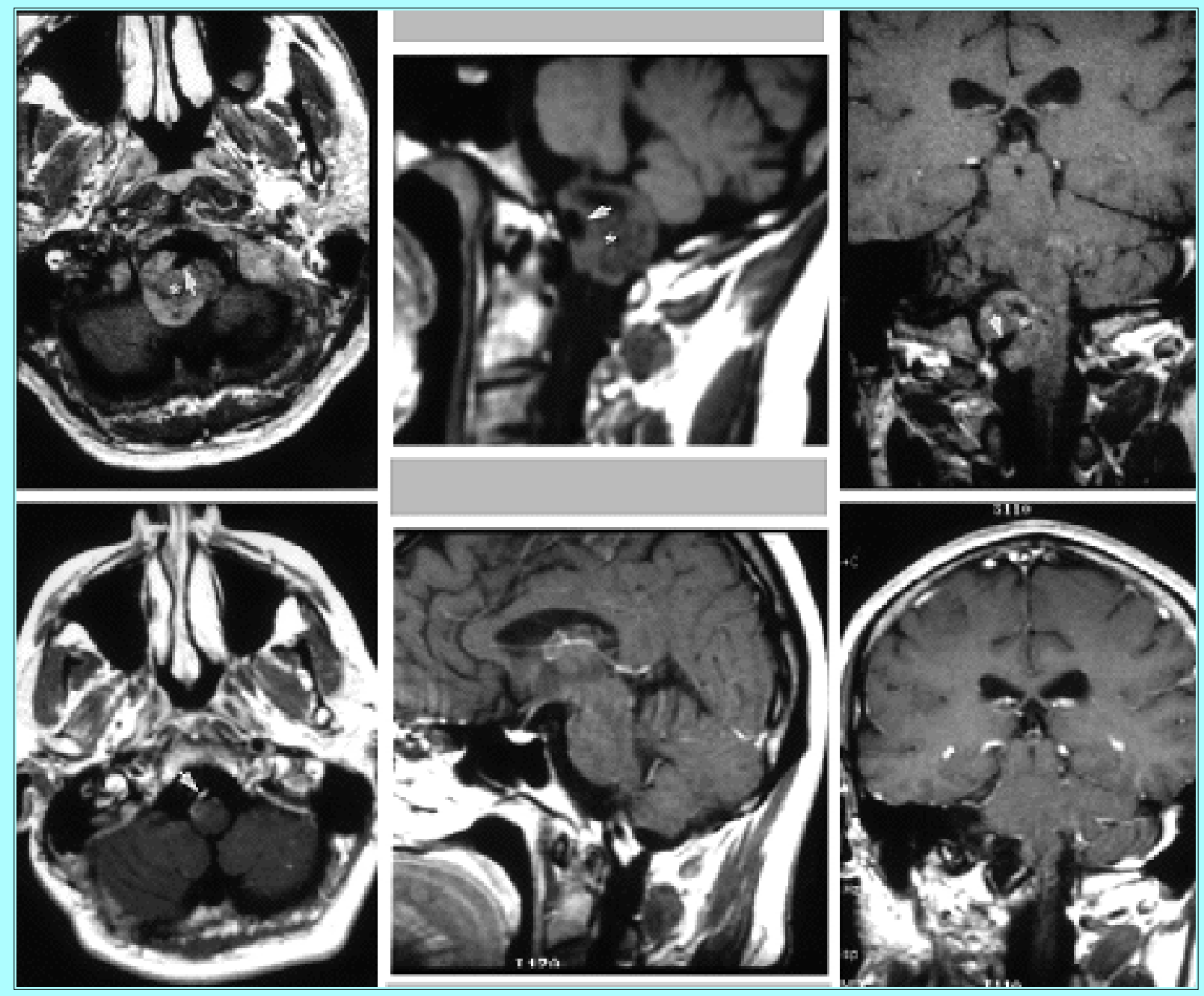

Fig. 7. Pre- and postoperative imaging studies. Upper: Preoperative contrast-enhanced MR images (left: axial; center: coronal; right: sagittal views) revealing a slightly hyperintense tumor $(*)$ encasing the VA (arrows). Lower: Postoperative contrast-enhanced MR images (left: axial; center: coronal; right: sagittal views) demonstrating a near-total tumor removal with a few-millimeter-thick residual cuff of the cauterized tumor left around the VA (arrows) because the arachnoidal plane could not be established between the two structures.

All but three patients underwent one-stage tumor removal. In these three patients, dissection of the SCS and transposition of the VA complex were difficult because of the significant engorgement of the SCS. Therefore, the intradural part of surgery was postponed 1, 2, and 5 days, respectively. In one patient, the tumor adhered to the VA and the 11th cranial nerve, and an arachnoidal plane could not be established. Our persistence in attempting to separate these structures led to rupture of the VA, which was repaired by using microsurgical sutures and then then reinforced with a Sundt clip. The 11 th cranial nerve in this patient was transected, dissected free, and reanastomosed.

\section{Surgery-Related Complications}

In addition to the six patients in whom preoperatively demonstrated palsy of the ninth and 10th cranial nerves worsened postoperatively, four other patients developed cranial nerve palsy after surgery. Of these 10 patients, two underwent vocal cord medialization and in one the vocal cord was injected with Gelfoam. Two patients underwent a tracheostomy, and in four a gastric tube was placed. The longer periods of hospitalization of these patients contributed to an overall increased average length of hospital stay. Eventually, all patients except one, who died during the follow-up period, recovered or 
compensated to a functional swallowing function, progressing from a dysphagic diet to a regular diet within 8 months. Of the two patients with 11th cranial nerve palsy, one was the patient in whom transsection and reanastomosis of the cranial nerve were performed. Both patients recovered to the preoperative level of function. Seven patients developed a 12th and one patient a sixth cranial nerve palsy; in all these patients resolution was complete except in two patients who experienced only a partial recovery.

Four patients developed a cerebrospinal fluid (CSF) leak. Two of these patients received additional skin sutures, whereas the other two were treated with external lumbar drainage and operative revision. One patient developed hydrocephalus that required placement of a shunt.

\section{Clinical Outcomes}

The overview of patients and clinical outcomes is presented in Table 2. All but two patients (Cases 9 and 12; KPS scores of 30 and 40, respectively) presented with a KPS score of 60 and higher (89\%). In all but three patients (83\%) an improved KPS score was obtained during the follow-up period compared with that obtained preoperatively; this improvement (Table 2$)$ was statistically significant $(\mathrm{p}<0.0001)$. The difference was also statistically significant at 12,18 , and 24 months postoperatively $(\mathrm{p}<0.0001)$. The KPS score for three patients remained unchanged postoperatively. Overall, however, the improvement varied from scores of 10 to 30 . A KPS score improvement of 10 points was demonstrated (28\%; mean follow up 29 months), 20 points in seven patients (39\%; mean follow up 50 months), and 30 points in three patients (17\%; mean follow up 46 months). In patients who underwent near-total or subtotal tumor removal stable tumor size was demonstrated on yearly follow-up MR imaging studies (mean 64 months), except in one patient, who eventually died. An analysis in which Cox's proportional hazard model was used, revealed that radical tumor resection (gross total or near total, as defined previously; see Figs. 7, 8, and 9) and a higher preoperative KPS score had statistically significant impact on the favorable outcome in our series ( $p<0.03$ and $p<0.02$, respectively), whereas sex and, interestingly, age had no influence. 

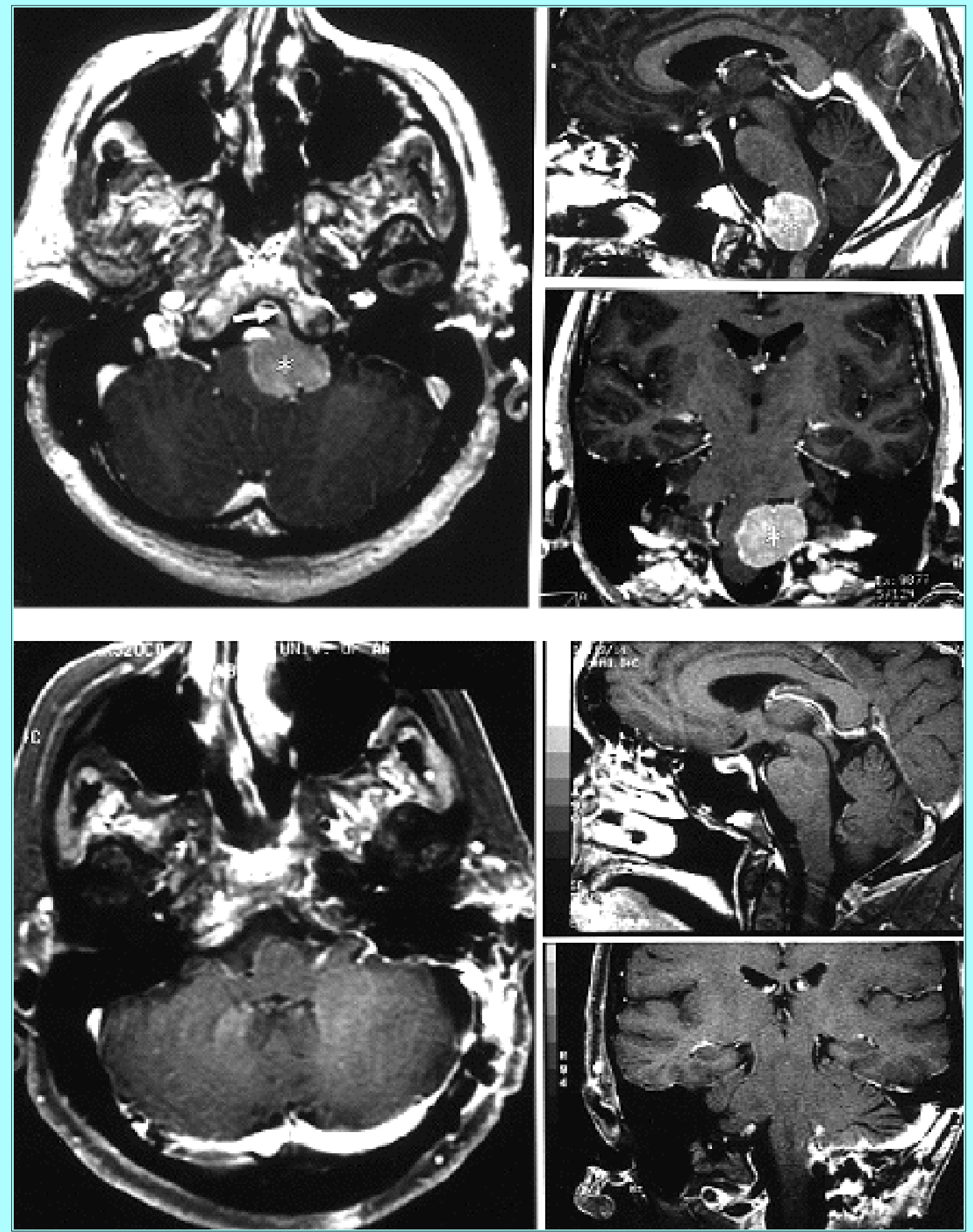

Fig. 8. Pre- and postoperative imaging studies. Upper: Preoperative contrast-enhanced $\mathrm{T}_{1}$-weighted images revealing the hyperintense tumor $(*)$ that compresses the cervicomedullary junction. Note the dural tail (arrow). Lower: Postoperative contrast-enhanced $\mathrm{T}_{1}$-weighted $\mathrm{MR}$ images revealing radical tumor resection. (Views in images: left: axial; upper right: sagittal; and lower right: coronal.). 


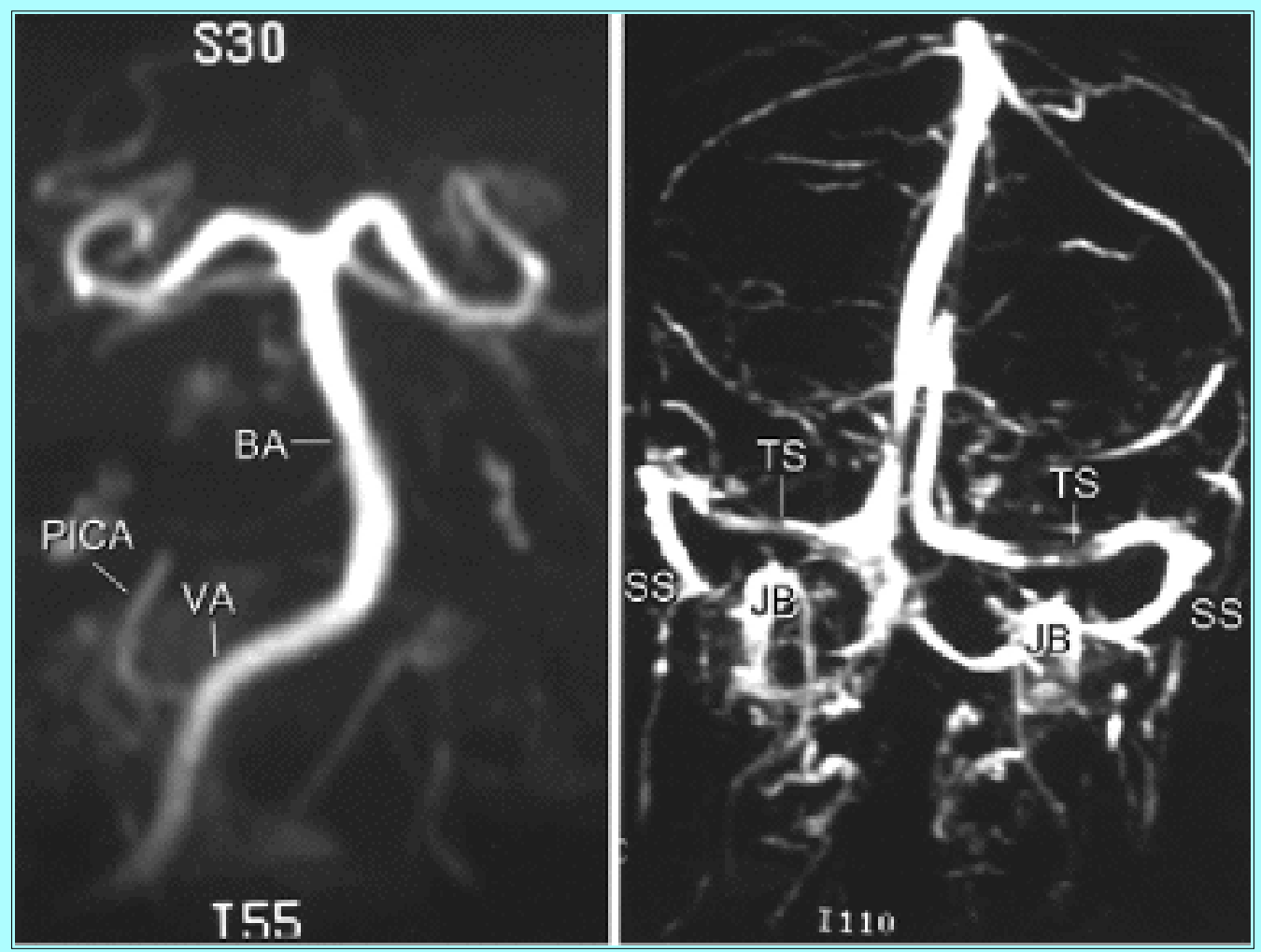

Fig. 9. The MR angiogram obtained in the same patient as in Fig. 8. At left, image obtained in the arteriography phase; note the basilar artery (BA), the right VA, the posterior-inferior cerebellar artery (PICA), and the aplasia (absence) of the left VA. At right, image obtained in the venous phase; note the bilateral transverse sinuses (TS), the sigmoid sinuses (SS), and the jugular bulbs (JB).

There were no perioperative deaths. Four patients died in the follow-up period (Table 2). In the first patient in this series (Case 1) a gross-total tumor removal was performed. She made a full recovery postoperatively, and her follow-up course was uneventful for 3 years until she developed multiple myeloma. She underwent radio- and chemotherapy but died 18 months later at the age of 79 years. One patient (Case 4) died more than 3 years after undergoing surgery for tumor recurrence. He presented with intractable facial pain, gait difficulties, ataxia, and diplopia caused by a sixth cranial nerve palsy, and he underwent subtotal resection that achieved satisfactory decompression of the nerve. The tumor was cauterized extensively at its origin, and the vascular supply interrupted as much as possible.

Postoperatively, he underwent radiotherapy, his symptoms resolved, and he returned to a normal life for 3 years, during which time a stable tumor size was demonstrated on the annual follow-up MR imaging. However, his symptoms recurred with worsening of his heart condition, gait difficulties, and swallowing problems, and the tumor had increased in size. The patient refused further therapy, his condition progressively worsened, and he died 3 weeks later at the age of 80 years. The third patient (Case 9) presented with severe quadriparesis and had difficulties with swallowing and diplopia. An aggressive postoperative course included placement of a gastric tube, early tracheostomy, and vocal cord medialization. She developed a CSF leak, which was surgically revised, followed by hydrocephalus, which required placement of a shunt. Her condition gradually stabilized, she was transferred to a rehabilitation facility and subsequently to a nursing home. At the 4-month follow-up examination, her quadriparesis had improved and she was ambulating with a walker. One month later, she was admitted to a local hospital for a deep venous thrombosis and received an inferior vena cava filter. Subsequently, she developed endocarditis and died 5 months after the surgery at the age of 64 years. The fourth patient 
(Case 12) who died in the follow-up period presented with quadriparesis, dysarthria, and swallowing difficulties. She also had a history of atrial fibrillation and myocardial infarction. Her status on postoperative neurological examination was unchanged. A gastric tube was inserted and a tracheostomy performed early, and she was transferred to a rehabilitation facility, where she developed a pulmonary embolus and died 1.5 months after the surgery at the age of 78 years.

\section{Histological Findings}

In this series, the most common histological meningioma type was meningotheliomatous. All but one patient underwent near-total or subtotal resection harbored this tumor type. The transitional type was slightly more common than psammomatous. The distribution of meningioma histological types is shown in Fig. 10.

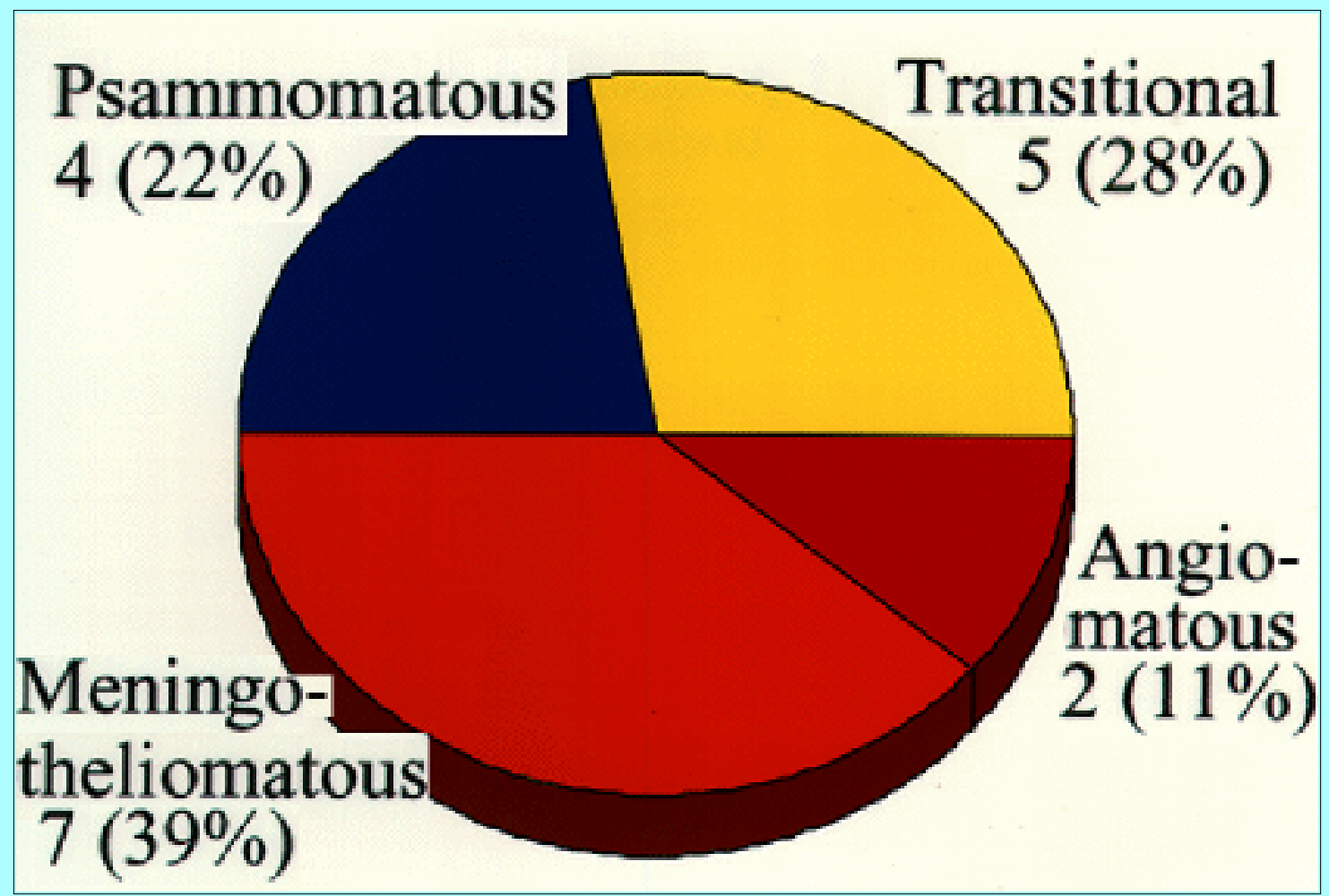

Fig. 10. Pie chart showing the distribution of meningioma histological types.

\section{DISCUSSION}

\section{Clinical Outcomes}

Because of their location, meningiomas located ventrally in the foramen magnum remain one of the most challenging meningiomas to treat. Often, they are not discovered until they are large, and the patient has experienced a relatively long period of minor or no symptoms. Their rare incidence $(<3 \%$ of all meningiomas) limits experience in their treatment. Yasargil, et al.,[39] reviewed 114 patients surgically treated for foramen magnum meningioma whose cases were reported between 1924 and 1976. They found an overall mortality rate of about $13 \%$, an overall "good" outcome of $69 \%$, a "fair" outcome of $8 \%$, and a "poor" outcome of $10 \%$. The Mayo clinic experiences were reported at three different times[14,26,40] and included all benign extramedullary tumors of the foramen magnum. Therefore, the operative results of isolated ventrally located tumors cannot be assessed.

Of six series on foramen magnum meningioma (that included those defined as located ventrally) reported 
in the most recent 15-year period,[5,15-17,19,22,30,33] four were from Europe.[15-17,19,22,30] Fourteen cases of meningioma of the ventral foramen magnum were reported by Guidetti and Spalone[19] (100\% total tumor removal; 14\% mortality rate), five by Sen and Sekhar[33] (60\% total tumor removal; 20\% perioperative mortality rate), seven by Kratimenos and Crockard[22] (86\% total tumor removal; 29\% perioperative mortality rate), and eight were reported by Babu, et al.,[5] (75\% total tumor removal, $13 \%$ mortality rate). The largest series of these lesions (39 cases) was reported by George, et al.,[17] who achieved 56\% of Simpson Grade I and 31\% of Grade II tumor resection, with an associated perioperative mortality rate of $8 \%$. Recently, Samii, et al.,[30] reported 40 cases of meningiomas located at the craniocervical junction (63\% total and 30\% subtotal tumor removal; 6\% perioperative mortality rates). This series probably included 25 cases of ventral foramen magnum meningioma, as defined herein. Unfortunately, the surgical results for this subgroup cannot be isolated.

Because our series deals only with ventrally located meningioma of the foramen magnum, comparisons with other series may be biased. Furthermore, it is difficult and, in part, scientifically inaccurate to compare series of patients in whom different variables exist (age, sex, exact tumor location, size, preoperative symptoms, meningioma type). Nonetheless, some conclusions can be drawn. As Fig. 6 illustrates, we were able to totally remove $75 \%$ of tumors at the first surgery; in the two surgical procedures performed to treat tumor recurrence, both removals were subtotal. This fact strongly suggests that radical tumor surgery should be pursued with zeal at initial surgery. As our results indicate, it is the best time to effect a "cure." A radical tumor resection proved to be a variable with a statistically significant impact on favorable clinical outcome. Conversely, the feasibility of radical resection cannot be predicted prior to surgery in patients presenting with a recurrent tumor, and gross-total resection is less likely to be achieved. Nonetheless, our results indicate that the surgeon should still attempt the maximum possible decompression and cauterization of the capsule and tumor origin. This strategy appeared to contribute to a relatively satisfactory quality of life (as indicated by KPS scores ranging from 60-90; mean KPS score 75) and a relatively long period in which the patient maintained a stable condition (mean 59 months). Furthermore, surgery itself was shown to have a statistically significant impact on the improvement of the follow-up KPS score compared with the preoperative score. Age and sex, on the other hand, were not shown to exert an impact on the outcome. Naturally, a longer follow-up period than the current mean of 40 months is necessary for confirmation of results.

Although no perioperative deaths occurred, four patients died during the follow-up period. Our first patient died of an unrelated disease (multiple myeloma). The second, in whom surgery was performed for a recurrent tumor, lived independently with a stable tumor, until it recurred again. Such a relatively long, stable follow up in this latter patient, along with that in the only other patient who underwent surgery for a recurrent tumor and who is still alive and in good condition, lends credence to an aggressive treatment in such cases. Two other patients (Cases 12 and 9) died 1.5 and 5 months, respectively, after surgery of causes not directly related to surgery (pulmonary embolus and endocarditis, respectively). Nonetheless, these cases must be analyzed closely with respect to the surgery. These two cases (aged 63 and 77 years) were actually the only patients in whom preoperative KPS scores were very low (Case 9, 30; Case 12, 40) and in whom progressive deterioration of their quadriparesis and swallowing problems were present. In fact, a low preoperative KPS score was a feature that had a statistically significant impact on the unfavorable outcome. The difficult decision to proceed with treatment was reached with these patients and their families. Although gross-total resection was achieved without technical difficulties and aggressive treatment (such as tracheostomy and gastric tubes) initiated postoperatively, both patients (particularly Case 9) experienced a series of complications. The conditions of both patients 
eventually stabilized, and they were transferred to a rehabilitation facility, with either minimally improved or an unchanged neurological status. Based on these cases, we learned that a low presenting KPS score, a progressive clinical course, and quadriparesis carry a poor prognosis and a significant risk. Hence, early diagnosis and operative treatment is recommended.

\section{Operative Techniques}

Treatment of meningioma of the ventral foramen magnum was begun by neurosurgical pioneers[10,12,14,18,19,38-40] who opened a door of what was once considered a "no man's land."[25] Traditionally, posterior midline suboccipital approaches and C-1 laminectomy have been used when treating these lesions.[38,39] Although the transoral approach $[9,27]$ has been advocated because it provides a direct route to the craniocervical junction, for intradural lesions it has serious limitations: poor access to laterally placed or broad-based tumors; crossing of a contaminated operative field (oropharynx) that causes meningitis or a CSF fistula; difficult dural closure; and destabilization of the craniocervical junction.[27] We found, as have other investigators,[5-7,15-17,22-25,30,33] that the transcondylar approach is greatly advantageous in the treatment of these lesions. Although a number of different terms have been used to describe this approach, there is in essence only one approach with variations in patient positioning, skin incision, muscle reflection, VA transposition, amount of condyle drilling, and craniotomy. It should, of course, be tailored to fit the local anatomy and tumor characteristics in each patient.

Our experience indicates that drilling of the condyle is very important for a safe and radical tumor resection. In our series, it ranged from approximately one-third to one-half of the condyle. This amount of drilling did not cause any craniocervical instability in our series. Another important factor, which cannot be predicted using present diagnostic modalities, is the size and development of the complex suboccipital venous compartment, the SCS. $[3,4,41]$ The SCS provides an alternative anastomotic route for intracranial venous return. In some patients, this sinus can be engorged because the tumor compresses the basilar and epidural venous plexi. Thus, the sinus becomes a troublesome source of bleeding and potential air embolism. This risk may lead to a prolonged, complex dissection and transposition of the VA complex cushioned in the SCS. For this reason, we performed the intradural component of surgery at a later stage in three of our patients. Hyperostosis associated with the meningioma of the ventral foramen magnum indicates tumor invasion.[28] Aggressive bone drilling of the hyperostotic site is recommended to prevent regrowth from this nidus and tumor recurrence.

\section{Perioperative Complications}

In our series, the most common complication was the palsy of lower cranial nerves (the ninth and 10th cranial nerves in particular). As other authors have also pointed out,[17,22,25,30,33] these cranial nerve palsies are the most dangerous. They have significantly contributed to the prolonged length of hospital stay in our series. Fortunately, in nearly all patients in our series function was recovered or compensated for within 8 months of surgery. We found it useful to try to predict its appearance and establish a preoperative baseline evaluation, including a formal, or at least bedside, swallowing study. This may have prevented the occurrence of aspiration, pneumonia, and possibly lethal outcome in our series. Postoperative treatment of these cranial nerve palsies should be cautiously administered and tailored according to the patient's circumstances. We advocate early placement of nasogastric or gastric tubes; ear, nose, and throat monitoring with vocal cord injections or medialization and tracheostomies as indicated. Postoperatively, a speech pathologist, present to monitor and adjust feeding and types of diet, 
is very beneficial. Nearly all patients recover from palsies of other (sixth, 11th, and 12th) cranial nerves as long as the nerves are anatomically preserved and were manipulated "cautiously" during surgery.

Four patients in our series developed postoperative CSF leak. Although watertight closure after transposition of the VA complex may be difficult, compulsive dural closure with fascial and fat autografts remains the only way to prevent this potentially dangerous complication. Nonetheless, there appears to be a learning curve associated with this complication because all cases in the present series occured in the early phase. In addition, hydrocephalus may be the underlying cause of the leak that requires shunting. If a CSF leak occurs, additional sutures and external lumbar drainage should be attempted first. If this approach fails, revision is warranted.

\section{Histological Findings}

The histological findings of meningioma of the foramen magnum have been reported only sporadically.[17,35,40] Although the meningotheliomatous type was most common in our series (39\%), it did not occur as frequently in reports by Yasuoka, et al.,[40] (68\%) and George, et al.,[17] (55\%). Although the psammomatous type was represented in only $22 \%$ of our patients, it occurred in the majority of cases reported by Stein, et al.[35] Although the histological type of meningioma may have had some as yet unknown impact on the degree of tumor removal (meningotheliomatous type was present in all but one patient in whom near-total or subtotal tumor resection was performed), it does not seem to have influenced the clinical outcome in our series.

\section{CONCLUSIONS}

In the majority of patients, meningioma located ventrally in the foramen magnum can be radically removed via the transcondylar approach, with a frequent but transient morbidity from lower cranial nerve palsies. Radical removal should be attempted during the first surgery because it is the best opportunity to effect a "cure." Radical removal of a recurrent tumor, although less likely to be achieved, is also recommended, because most patients live a relatively long, stable period of time in a good neurological condition. Patients with progressive motor weakness and a low preoperative KPS score have a poor prognosis. Hence, early diagnosis and operative treatment is recommended.

\section{Acknowledgments}

We are indebted to Ms. Julie Yamamoto for editorial assistance, Ms. Carolyn H. Thompson, M.S., for statistical analysis, and Mr. Ron M. Tribell for original artwork. We would like to thank Kevin Yoo, M.D., (Department of Neurosurgery, Loyola University), Alfred Bowles, M.D., and Ms. Kathy Suber (Department of Neurosurgery, University of Mississippi) for providing necessary data to complete this study. We are grateful to Ms. Sanja Arnautovic for tireless data gathering and processing.

\section{References}

1. Al-Mefty O: Operative Atlas of Meningiomas. Philadelphia: Lippincott-Raven, 1998, pp 249-382

2. Al-Mefty O, Borba LAB, Aoki N, et al: The transcondylar approach to extradural nonneoplastic lesions of the craniovertebral junction. J Neurosurg 84:1-6, 1996

3. Arnautovic KI, Al-Mefty O: Foramen magnum meningiomas, in Black PM, Kaye A (eds): Operative Neurosurgery. London: Harcourt Brace (In press, 1999) 
4. Arnautovic KI, Al-Mefty O, Pait TG, et al: The suboccipital cavernous sinus. J Neurosurg 86:252-262, 1997

5. Babu RP, Sekhar LN, Wright DC: Extreme lateral transcondylar approach: technical improvements and lessons learned. J Neurosurg 81:49-59, 1994

6. Baldwin HZ, Miller CG, van Loveren HR, et al: The far lateral/combined supra- and infratentorial approach. A human cadaveric prosection for routes of access to the petroclival region and vertebral brain stem. J Neurosurg 81:60-68, 1994

7. Bertalanffy H, Seeger W: The dorsolateral, suboccipital, transcondylar approach to the lower clivus and anterior portion of the craniocervical junction. Neurosurgery 29:815-821, 1991

8. Blom S, Ekbom KA: Early clinical signs of meningiomas of the foramen magnum: a new syndrome. $\mathbf{J}$ Neurosurg 19:661-664, 1962

9. Bonkowski JA, Gibson RD, Snape L: Foramen magnum meningioma: transoral resection with a bone baffle to prevent CSF leakage. Case report. J Neurosurg 72:493-496, 1990

10. Castellano F, Ruggiero G: Meningiomas of the posterior fossa. Acta Radiol (Suppl) 104:1-164, 1953

11. Cohen L, Macrae D: Tumors in the region of the foramen magnum. J Neurosurg 19:462-469, 1962

12. Cushing H, Eisenhardt L: Meningiomas. Their Classification, Regional Behaviour, Life History and Surgical End Results. Springfield, Ill: Charles C. Thomas, 1938

13. de Oliveira E, Rhoton AL Jr, Peace D: Microsurgical anatomy of the region of the foramen magnum. Surg Neurol 24:293-352, 1985

14. Dodge HW Jr, Love JG, Gottlieb CM: Benign tumors at the foramen magnum. J Neurosurg 13:603-617, 1956

15. George B, Dematons C, Cophignon J: Lateral approach to the anterior portion of the foramen magnum. Application to surgical removal of 14 benign tumors: technical note. Surg Neurol 29:484-490, 1988

16. George B, Lot G: Anterolateral and posterolateral approaches to the foramen magnum: technical description and experience from 97 cases. Skull Base Surg 5:9-19, 1995

17. George B, Lot G, Boissonnet H: Meningioma of the foramen magnum: a series of 40 cases. Surg Neurol 47:371-379, 1997

18. Guidetti B, Spallone A: Benign extramedullary tumors of the foramen magnum. Surg Neurol 13:9-17, 1980

19. Guidetti B, Spallone A: Benign extramedullary tumors of the foramen magnum. Adv Tech Stand Neurosurg 16:83-120, 1988

20. Haddad GF, Al-Mefty O: Infratentorial and foramen magnum meningiomas, in Wilkins RH, Rengachary SS (eds): Neurosurgery, ed 2. New York: McGraw-Hill, 1996, Vol 1, pp 951-958 
21. Karnofsky DA, Burchenal JH: The clinical evaluation of chemotherapeutic agents in cancer, in MacLeod CM (ed): Evaluation of Chemotherapeutic Agents. New York: Columbia University Press, 1949, pp 191-205

22. Kratimenos GP, Crockard HA: The far lateral approach for ventrally placed foramen magnum and upper cervical spine tumours. Br J Neurosurg 7:29-140, 1993

23. McDermott MW, Wilson CB: Meningiomas, in Youmans JR (ed): Neurological Surgery, ed 4. Philadelphia: WB Saunders, 1996, Vol 4, pp 2782-2825

24. Menezes AH, Traynelis VC: Tumors of the craniovertebral junction, in Youmans JR (ed):

Neurological Surgery, ed 4. Philadelphia: WB Saunders, 1996, Vol 4, pp 3041-3072

25. Menezes AH, Traynellis VC, Gantz BJ: Surgical approaches to the craniovertebral junction. Clin Neurosurg 41:187-203, 1993

26. Meyer FB, Ebersold MJ, Reese DF: Benign tumors of the foramen magnum. J Neurosurg 61:136-142, 1984

27. Miller E, Crockard HA: Transoral transclival removal of anteriorly placed meningiomas at the foramen magnum. Neurosurgery 20:966-968, 1987

28. Pieper DR, Al-Mefty O, Hanada Y, et al: Hyperostosis associated with meningioma of the cranial base: secondary changes or tumor invasion. Neurosurgery 44:742-747, 1999

29. Russell JR, Buery PC: Meningiomas of the posterior fossa. Surg Gynecol Obstet 96:183-192, 1953

30. Samii M, Klekamp J, Carwalho G: Surgical results for meningioma of the craniovertebral junction. Neurosurgery 39:1086-1095, 1996

31. Scott EW, Rhoton AL Jr: Foramen magnum meningiomas, in Al-Mefty O (ed): Meningiomas. New York: Raven Press, 1991, pp 543-568

32. Scott M: The surgical management of meningiomas of the cerebellar fossa. Surg Gynecol Obstetr 135:545-550, 1972

33. Sen CN, Sekhar LN: An extreme lateral approach to intradural lesions of the cervical spine and foramen magnum. Neurosurgery 27:197-204, 1990

34. Simpson D: The recurrence of intracranial meningiomas after surgical treatment. J Neurol Neurosurg Psychiatry 20:22-39, 1957

35. Stein BM, Leeds NE, Taveras JM, et al: Meningiomas of the foramen magnum. J Neurosurg 20:740-751, 1963

36. Welling B, Al-Mefty O: Foramen magnum tumors, in Cohen AR (ed): Surgical Disorders of the Fourth Ventricle. Cambridge, Mass: Blackwell Science, 1996, pp 251-261

37. Welling B, Park Y K, Al-Mefty O: Primary extramedullary tumors of the craniovertebral junction, in Dickman CA, Spetzler RF, Sonntag VKH (eds): Surgery of the Craniovertebral Junction. New York: Thieme, 1998, pp 239-252 
38. Yasargil MG: Microneurosurgery. Stuttgart: Thieme, 1996, Vol IVB, pp 178-179

39. Yasargil MG, Mortara RW, Curcic M: Meningiomas of basal posterior cranial fossa. Adv Techn Stand Neurosurg 7:1-115, 1980

40. Yasuoka S, Okazaki H, Daube JR, et al: Foramen magnum tumors. Analysis of 57 cases of benign extramedullary tumors. J Neurosurg 49:828-838, 1978

41. Zolnai B: Die zwischen der Arteria vertebralis und den vertebralen und zerebralen Venen bestehende Verbindung am atlantookzipitalen Abschnitt beim Menschen. Anat Anz Bd 114:400-407, 1964

Manuscript received April 22, 1999.

Accepted in final form May 14, 1999.

Address reprint requests to: Ossama Al-Mefty, M.D., F.A.C.S., Department of Neurosurgery, 4301 West Markham, Slot 507, Little Rock, Arkansas 72205. email: almeftyossama@ exchange.uams.edu. 